\title{
Sustainable solar power from wall mounted photovoltaics
}

\author{
M. S. Osigbemeh ${ }^{1}$ (1) O. S. Asaolu ${ }^{2}$. A. N. Nwachukwu ${ }^{3}$
}

Received: 7 August 2021 / Accepted: 19 November 2021

○ The Author(s), under exclusive licence to Springer-Verlag GmbH Germany, part of Springer Nature 2021

\begin{abstract}
The plausibility of wall-mounting of photovoltaics in inaccessible or restricted rooftops to generate power necessitated this study. Meeting energy consumption demands is an infrastructural challenge in several developing economies. Power generation could leverage on the photoelectric effect from intense diffuse radiation and intermittent direct solar radiation abundantly available in tropical Africa, near the equator. A test-bed was developed and an investigation was conducted into the energy consumption needs of small and medium scale buildings; namely, offices and small homesteads. The prototype test-bed was found to provide all the power requirements of an average consumer utilizing less than 5000 Watts daily load from the Sun's conventional daily migration from east to west. Increase in generated and consumed Wattage has been observed to increase with scalability of photovoltaics participating in the east, front and west wings of test-bed. The spatial analysis of the trajectory of solar energy for both directed and diffused intense solar radiation was also carried out in this work. The need of blocking power leaks to dormant photovoltaics due to conduction through less resistance pathway by active photovoltaics, via diode compensation or relay blocking was also discovered to increase the overall power generated that was available to the interconnected loads.
\end{abstract}

Keywords Wall-mount Photovoltaic · Mounting Solar panels · Power loss blocking · Diffuse radiation $\cdot$ Diode compensation $\cdot$ Blocking power leaks $\cdot$ Renewable energy

M. S. Osigbemeh

eosigbemeh@yahoo.com

1 Department of Electrical and Electronic Engineering, Alex Ekwueme Federal University, Ndufu-Alike, Nigeria

2 Department of Systems Engineering, University of Lagos, Lagos, Nigeria

3 Department of Physics, Alex Ekwueme Federal University, Ndufu-Alike, Nigeria 


\section{Introduction}

In resource constrained sub Saharan Africa, power needs continue to witness unprecedented challenges and bottlenecks in generation, transmission, distribution and consumption of grid-based energy to an increasing population density and necessitating dependence on largely non-grid-based solar power for energy demands. With proliferation of rooftop photovoltaics mounts, solar power has become readily available to small and medium scale consumers even in remote or inaccessible locations, allowing for off-grid power generation and consumption. This reduces dependence on utility companies especially in resource-constrained sub Saharan Africa. These photovoltaics possess abundant and, vibrant nearly allyear round solar radiance with some topological locations experiencing brilliant sunlight for more than thirteen hours a day to fulfill the renewable energy needs of connected devices. Consumers depending on photovoltaics power alone with stored energy in batteries backup at night times achieve appreciable power supply for a pre-computed energy consumption on a daily basis, with the normal movement of the Sun from conventional east to west. This is especially true around the equator for consumers living in cities with photovoltaic mounts at strategic positions. The average sunlight radiance in most parts of Africa reaches as much as $13 \mathrm{~h}$ of the usual $24 \mathrm{~h}$ daily sunshine in accordance with Burgess [6] and NASA [23]. With adequate consumer load demand calculation, solar power can be designed to be utilized for this period while still being stored through backup batteries for night use on a daily basis for each consumer homestead.

According to Burgess [6], the amount of direct radiation and the amount of diffuse radiation coming into the earth each day are about $75 \%$ of the energy coming from the sun which scientists have placed at a temperature of $5500^{\circ} \mathrm{C}$ with about 1370 Joules $(\mathrm{J})$ of energy reaching earth's atmosphere and about $1000 \mathrm{~J}$ reaching the earth's surface. The work reported that about $26 \%$ of the total sun radiation also experiences atmospheric absorption and scattering. This has necessitated the design of economical, environmentally friendly and socially sustainable photovoltaics that taps the enormous renewable energy. Such solutions are gradually reducing the dependence on fossil based power generation systems with its attendant greenhouse effects.

Becker [5] states that "direct solar radiation (I) is the proportion of the almost rectilinear solar radiation, which reaches the earth's surface from an angle with a distance of $0.25^{\circ}$ to the center of the sun and reaches a normal area, which is oriented perpendicularly to the direction of the radiation." The source also stated that diffuse radiation is the portion of solar radiation which arrives on the surface of the earth after single or repeated dispersion in the atmosphere with a radiation intensity of the diffuse radiation depending on the variable position of the sun.

Extensive research has been carried out on the abundance of global solar irradiance (GSI) in tropical regions in Africa such as Nigeria which is located between $4^{\circ} \mathrm{N}$ and $14^{\circ} \mathrm{N}$ latitude. Nigeria has a vast supply of solar energy all year round according to Osinowo et al. [26]. The enormous radiance available from the sun has been analyzed by Iqbal [16], Illera et al. [17] and Osinowo and Okogbue 
[25] and the seemingly harsh radiation and its effect on earth's surfaces has been enumerated by Radosavljevic and Dordevic [27] and Hussien [20]. The dependence of solar radiation maps for analyzing solar intensities have been investigated by Halouani et al. [14] and Linares-Rodríguez et al. [19] thus aiding designers of solar energy systems to more efficiently utilize solar irradiance for profitable uses.

Investigations on the increased intensities of the Sun's radiation in tropical climate towns along or close to the equator in Africa has been carried out by Akpabio and Etuk [1]; Akpabio et al. [2]). It was reported that the increased intensities along the equator within tropical climate locations were expected since the earth is a huge spinning and revolving sphere in which towns around the equator are closest to the Sun. Their experimental observation is buttressed with the inverse square law for light intensity, which states that the intensity of solar radiation at any point in the solar system (earth inclusive) is inversely proportional to the square of that point's distance from the Sun.

The computation of the intensity of solar radiation or solar irradiance with respect to any point on the surface of the earth according to Engineering 360, [11] can be computed from the relation:

$$
I \approx \frac{c n \epsilon^{\epsilon}}{2}|E|^{2}
$$

where $\mathrm{c}=$ speed of light within a vacuum; $E=$ complex attitude of the wave's electric field; $n=$ refractive index of the surface and $\epsilon^{\epsilon}=$ vacuum permeability.

Osinowo et al. [26] also generated annual and seasonal solar irradiance maps for Nigeria with a fractional distribution and its coefficient of variation over the various climatic zones from a 26 year (1984 - 2009) daily satellite global irradiance data. The analysis depended on archived data from NASA and validational data for the surface global irradiance (SGI) data obtained from the Nigerian metrological Agency (NIMET) for the period of the analysis. The author stated that daily clearness index (DCI) ranged between 0.63 and 0.43 for all locations in Nigeria while GSI ranged between $21.63 \mathrm{MJ} / \mathrm{m}^{2} /$ day (mega joules per meter square per day) and $11.95 \mathrm{MJ} / \mathrm{m}^{2} /$ day during the summer months of June - September. Lower values for the SGI and DCI where observed during the winter periods of November - February according to the source when compared to the average annual spatial pattern in corresponding location. They concluded that "peak global solar irradiance and clearness index are seen at the Northern horn of the maps with steady decrease in surface receipt southwards. Not much spatial variation in surface irradiance and clearness index were however observed between the seasons but little differential changes in magnitude were observed in Northern and Southern Nigeria". The North-South differential was attributed to seasonality in cloudiness and the solar angle tilt of studied locations.

Improvements in photovoltaics design and construction that lead to very efficient and reliable solar panels has allowed for a wider conduction period for photovoltaics systems depending on the sun's energy or its radiant light through the utilization of the photoelectric effect [ $4,13,22]$. These devices which are classified mainly 
as mono $\mathrm{Si}$ and poly $\mathrm{Si}$ are known to conduct and produce appreciable power even at extremely low solar intensities and thresholds. This notable improvement has subsequently allowed the design of power storage systems which allows for continuous power for loads all through the $24 \mathrm{~h}$ of 7 days. Solar photovoltaic systems continue to experience massive deployments even for very large scale electrification and power supply needs in highly developed countries where smart technologies and control are been used to integrate the generated power unto on-grid support systems for energy consumption by consumers. However, these deployments are characterized with large mass or area grabbing and the effect of adverse or excessive winds, animal interferences such as herds, birds, etc. Other debilitating factors in tropical regions include the need for constant site maintenance from over-grown vegetation like trees, grasses, etc.

With advancements in thin film technologies and large scale automated system integration of manufacturing and production processes, photovoltaics have witnessed tremendous reductions in size, costs and deployments. Far back as 2010, Germany was reported by the International Renewable Energy Agency [31] of having the lowest photovoltaic system costs and maintenance in the small-scale residential market $(<5 \mathrm{~kW})$ at an average of USD 3.8/W for c-Si systems. This pricing further experienced a dip, at the second quarter of 2012, to just USD 2.2/W. In resource-constrained African economies, solar power demand continues to rise for medium and small scale private homes or consumers. This is attributable to the unavailability of useful power from grid-based utility companies and their unaffordable charges. However, due to the COVID-19 pandemic with consequences that included shutting down of economics and most manufacturing outfits in China, a sharp rise in the pricing of this photovoltaics have been witnessed. For instance, a $200 \mathrm{~W}$ mono solar panel was sold as much as USD 100 in Lagos, Nigeria after the COVID -19 national lockdown. Thus, the increasing cost of solar panels has caused a reduction in the rapid growth of solar deployments in Africa despite the population explosion in the continent. A rising complementary problem in sub-Saharan Africa is the need for accessible rooftops on which photovoltaics could be attached for the generation and consumption of this renewable energy. These constraints and requirements have necessitated considerations of wall mounts for mounting of photovoltaics for solar power optimizations.

Problems like tracking the pathway of the conventional migration of the sun and its intense radiation also known as solar irradiance was achieved using the solar irradiance instrument called pyranometer and by physical observances. Solar irradiance according to Finsterie et al. [12], The World bank [32] and Alternative Energy [3] is the solar intensity or the power per unit area received from the sun in the form of electromagnetic radiation as measured in the wavelength range of the measuring instruments in watt per square meters $\left(\mathrm{W} / \mathrm{m}^{2}\right)$ or the amount of solar energy per unit area arriving on a surface of the earth at a particular angle with respect to the sun. Another problem is the photovoltaics' inclination to the sun's trajectory for optimum energy harness on daily basis. In a research that involved a model for estimating the average global solar radiation of a tropical climate town in West Africa using data on sunshine duration, mean temperature, relative humidity, number of rainy day(s), wind speed, etc. obtained from the Nigerian Meteorological Agency for a period 
of eleven years (1997 to 2007), by Umoh et al. [33] it was observed that multiple regression analysis could be used to develop correlation equations capable of detecting the greatest and least amounts of global solar radiation. A sum of the real time radiation from direct and diffuse solar radiations is known as global solar radiation and sometimes referred to as "sun + sky" radiation as stated by Engineering360, [11].

The plausibility of attaining significant and sustainable power from wall mounted photovoltaics for powering small and medium scale consumers is the major contribution of this research work. The structure of this paper is hinged on a methodology that combines a five-year daily experimental and empirical investigation with experiences in the deployment and utilization of photovoltaics. Various power compensation for improved optimization of available power to photovoltaics by the authors was also investigated. Results and analysis of the various operating modes of the resulting sustainable and renewable energy sourced from photovoltaics has also been investigated. The paper concluded that significant powering of consumer terminals can be achieved by wall-mounted photovoltaics alone and also by a combination with rooftops photovoltaics and even in a grid based scheme.

\section{Materials and methods}

To attain appreciable power dissipation from wall mounts for consumer needs, experiments to determine the magnitude and direction of intense solar radiation for the daily direct component and diffuse component of radiated sunlight was conducted. The direct tracking approach and use of the solar irradiance instrument called pyranometer was used to obtain solar intensity readings per minute and on a daily basis. Validational data was obtained from archived and expected readings from literature per investigated location. An East wing corresponding to the area of wall mounted photovoltaics that are first to receive solar irradiance and hence produce power after dawn was established. These set was referred to as pvA and were responsible for powering attached load from early morning solar irradiance by a combination of directed and diffused solar irradiance. Also, a set of photovoltaics called pvB were mounted at the building's front or north side and a final set of photovoltaics were wall mounted at the west wing and named $\mathrm{pvC}$ as shown in Fig. 1. The arrangement was designed in such a manner to allow for the maximum achievable power from the solar irradiance available on a daily basis and all year round based on the extreme fact that apart from the rooftops of buildings, direct solar irradiance reaches at most three sides of a building daily while diffuse solar irradiance reaches all parts of the building. Each set of photovoltaics were arranged to obtain 400 watts each for charging five parallel connected deep cycle solar battery bank from Trillion Energy (120 watts each). The bank forms a total of 600 $\mathrm{AH}$ and utilizes the absorbent glass mat (AGM) and gel based cell technologies for valve regulated lead acid batteries (VRLA). By observing three charging sequence obtained from the optimization system designed, continuous power was achieved at attached load terminals during the duration of daily direct solar irradiance. Since the roof tops experienced largely direct solar irradiance and some form of global 


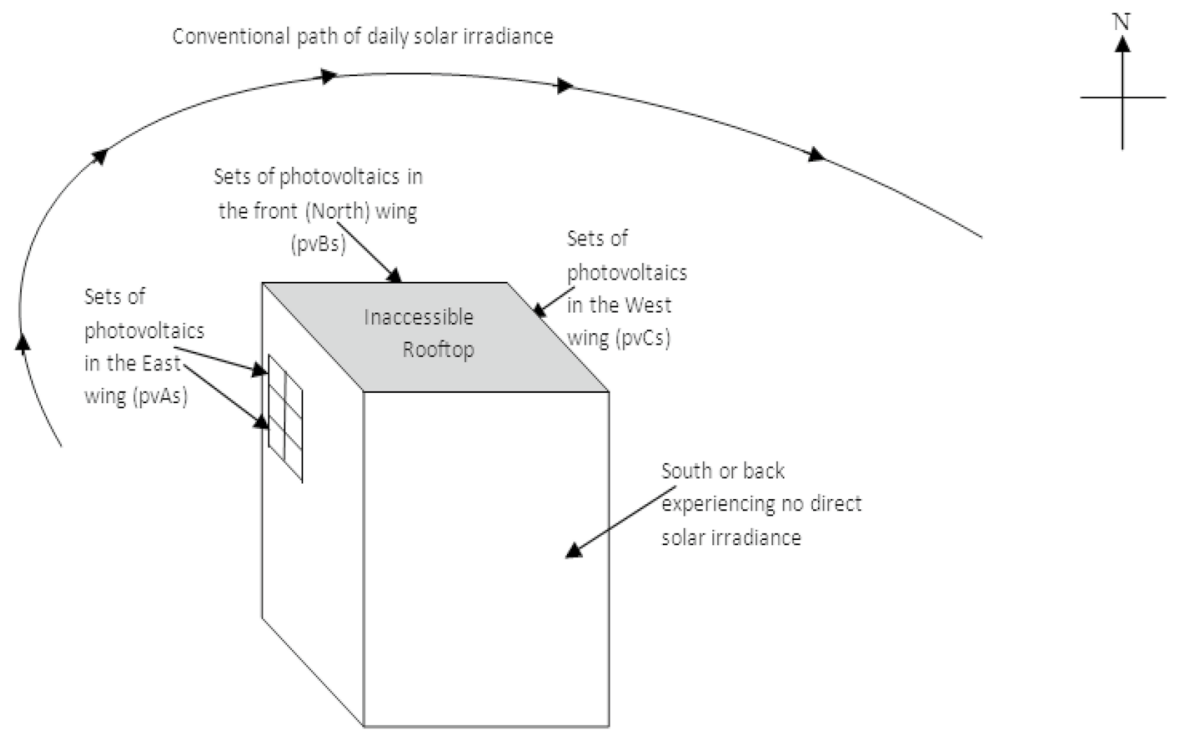

Fig. 1 Building with wall-mounted photovoltaics along irradiated wings

diffused radiation, it was not considered in this analysis. This is due to the fact that at all times the solar intensities at the roof top is always higher when compared with wall mounted photovoltaics especially when solar irradiance is directly overhead of building. The resulting optimization process of harnessing solar energy from wall mounted photovoltaics has been largely based on heuristic and results obtained from this work has been observed to be applicable and obtainable for any building's position provided that the three wings are pre determined with respect to the conventional movement of the sun and its trajectory in that location. Thus photovoltaics installed in the front wing of a particular building may be corresponding to the back or south wing of another building based on the buildings topology with respect to the daily trajectory of the sun. As part of the materials use for this research, it was ensured that participating photovoltaics where from the monocrystalline type over the polycrystalline photovoltaics and the thin film solar panels due to the fact that the monocrystalline photovoltaics provide more power than the polycrystalline and the thin film given the same solar intensities and durations during operation and performances. This was hinged from SolarReviews [29], Energysage [10] and Solar Magazine [28] and the photovoltaics where mounted at a height of from twenty feet from the ground for maximum and optimal solar energy harness.

At late mornings, pvBs experienced their maximum solar irradiance from mainly diffused radiation and intermittent direct radiations while pvAs are now experiencing only diffused radiation and pvC are yet to conduct. Intermittent solar irradiance was observed due to the non-linear and stochastic trajectory of solar irradiance. A spatial distribution of the study or observance of the trajectory followed by the sun when viewed from a residential building or test site positioned on earth showed a non-linear path in the Sun's daily migration from East to West by convention i.e. 


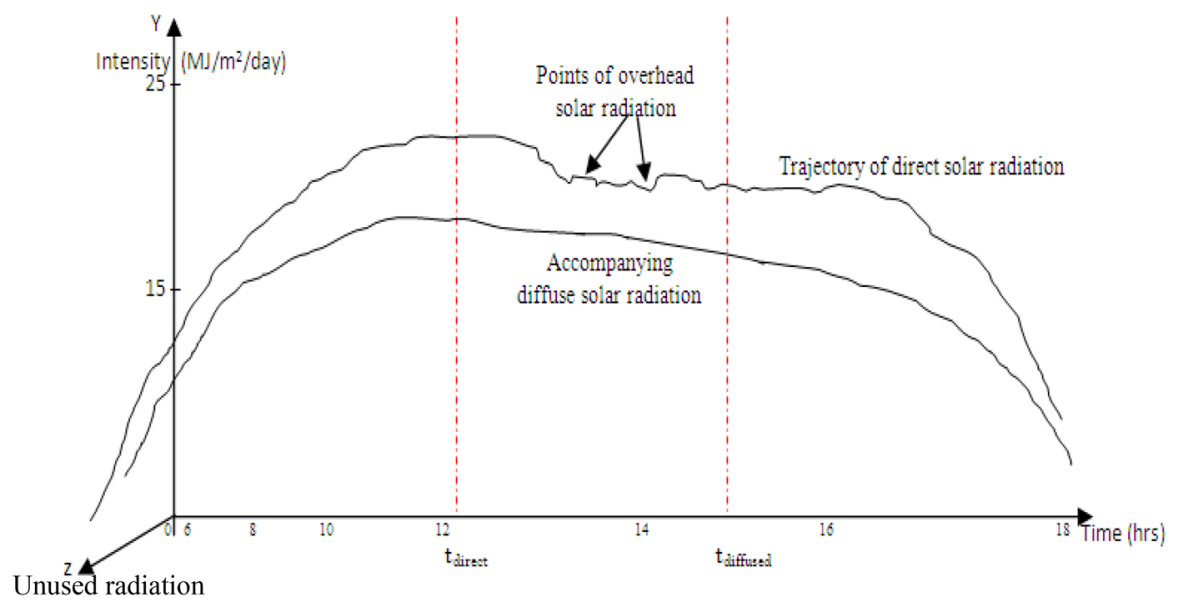

Fig. 2 Overall daily energy dissipation of all participating photovoltaics

its daily rise and fall or setting as observed. A depiction of the non-linear trajectory embarked by the Sun's irradiance or solar intensity over an average of the daily recording in two separate locations in Abakaliki, Ebonyi state and Lagos for the period of January 2016 through December 2020 is shown in Fig. 2.

From Fig. 2, it can be seen that solar irradiance as observed from test site increases sharply up to around noon when the sun is directly overhead of building or test site, i.e. at maximum irradiance, $t_{\text {direct }}$. At this period before noon or $t_{\text {direct }}$, mean power for consumer consumption is provided by set of photovoltaics in the east wing only which are experiencing both direct and diffused solar intensities. As solar irradiance begin to migrate to the west, depending on the stochastic nature of sun's trajectory, the front or north wing photovoltaics which have been experiencing increased diffused irradiance begins to experience increase in direct solar irradiance thus improving their conduction and power contribution to consumer terminals. At periods further than $t_{\text {difused }}$, corresponding to around $2-3 \mathrm{pm}$ on average; a steady conduction of the east wing photovoltaics ensues leading to mean power to consumer terminals largely form the direct and diffused solar irradiance hitting the east wing photovoltaics, pvC. The z-axis corresponds to the period of unused radiation from the very early morning solar radiation due to the very weak solar intensities and also not hitting or reaching east wing photovoltaics.

\subsection{Power compensation}

In the design, it was observed that useful power that would have sustained consumer loads flows through pvB and $\mathrm{pvC}$ since the sets of photovoltaics on the west and front wing provided path for least resistance when compared with the interconnected loads as power is been generated by pvAs. In other for the designed system's attached load not to experience power loss due to power leaking through the low resistance paths provided by the yet to conduct photovoltaics at the west and front 


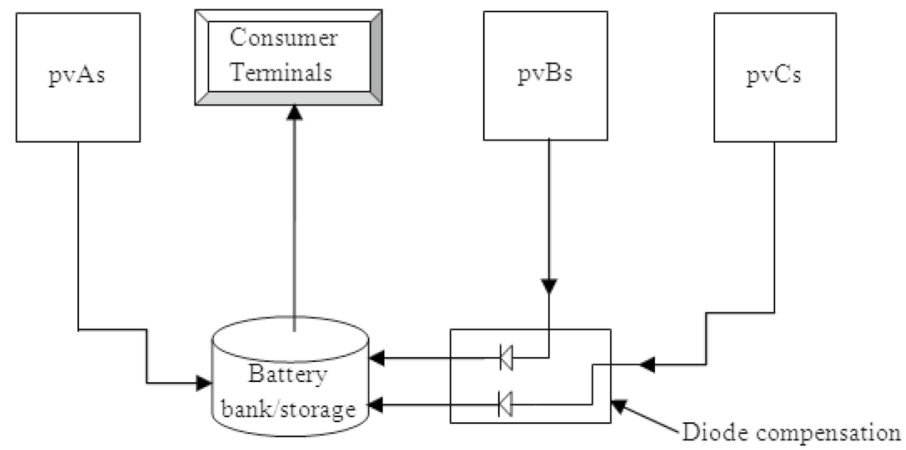

Fig. 3 Block diagram showing the power compensation connection

wings i.e. through pvBs and pvCs, a power compensation diode was introduced. The resulting power blocking circuitry which was connected in series and in reverse bias mode with pvBs and pvCs to block this power leak forced the hitherto leaked power to become available to applied load. See block diagram in Fig. 3.

\subsubsection{Diode / relay compensation}

As solar intensity approach optimality leading to appreciable power rise, blocking diode for pvBs begins conduction and allows current from the receiving solar radiation photovoltaics pvBs to supply and contribute to the resultant power available to the consumer terminals. It was observed that diffuse radiation played a major role for keeping the pvAs (now experiencing no direct solar irradiance) in conduction band thus preventing the need for connecting pvAs with blocking diodes as pvBs operate and pvCs yet to conduct. In the third and final charging sequence, from the Sun's pvCs located in the West wings, a sustained conduction begins to conduct after its power compensation diode has experienced sufficient power to become forward bias by leveraging on the intensity of both direct and diffuse solar irradiance. At that point, pvAs are on completely diffused radiation while pvBs and pvCs are experiencing both receding direct and diffused solar irradiances. The diode specification is 6A10-T MIC silicon rectifier diode chosen for its maximum DC blocking voltage, RMS voltage, recurrent peak reverse voltage, high surge current capability, etc. according to Diodes Incorporated [8].

It is important to note that during a complete overhead of solar radiation position of the sun, all participating photovoltaics were not experiencing direct solar radiation but only sustained power production from diffused radiation. Exceptions where noted for some intermittent direct solar irradiance when the sun was directly overhead based on sometimes stochastic movement of the sun and layout of building. At point of direct solar irradiance, power critical installation needing constant powering would require more attention or alternative power support from other means such as from roottops, grid-based, wind energy, etc. or power supply compensation for larger power consumption needs. In other to achieve a parsimonious and robust design, participating photovoltaics must 
provide appreciable power from diffuse radiation only during periods of solar overhead, a period in which diffuse radiation is at its highest. The dependence on the more efficient maximum power point tracking (MPPT) DC - DC solar converter as against the pulse width modulation controller (PWM) according to $[,, 7,18,34]$ is crucial in other to achieve optimal power from the non-linear trajectory of solar irradiance on daily basis for these wall mounts. The property of the blocking provided by the diode during reverse biasing was used to disconnect photovoltaics that were awaiting their turn to conduct or receive solar radiation in a medium size solar system. This was based on the fact that the site used for this research was pre-analyzed for the normal direct and diffuse radiation on daily basis with considerations for periods of extreme low solar radiation. The volt ampere characteristics of the diode of not allowing high voltages of about 50 volts to flow during reverse bias Hughes et al. [15] and Makarov et al. [21] was used to create a blocking off of current or power that would have followed the part of least resistance and flow through the not yet conducting photovoltaics.

Sharp drops in the direct and diffuse radiations due to sudden weather precipitation such as rainfall, hail, etc. leading to rapid loss of ambient temperature, pressure and humidity were considered. Such intermittent drops caused participating pvAs to lose charges rapidly thus, creating a low resistance path of power loss from pvBs within the system as a whole. Due to the shortness of the period of such sudden change with respect to normal daily solar radiation, this intermittent occurrence was ignored as little can be done to mitigate the adverse and short period. Ignoring this occurrence is justified since the blocking diode experiences a voltage drop when in use and operating all-day leading to significant undesirable power consumption and loss to load terminals. This is also hinged on the fact that considerable battery supply for charging would be affected by another blocking diode across pvAs. The alternative use of the mechanical relay when using the normally not connected (NNC) terminal when power is low through the East wing photovoltaics resulted in the same function with the diode compensation. The normally connected terminal is not left unconnected while connection to the load is via the NNC terminal. The NNC terminal connects the East wing photovoltaics to the load when a particular voltage threshold is reached by the solar intensity. However, additional circuitry and power consumption is needed to ensure proper usage of the relay as a compensator for power leakage. Thus when comparing the diode to the mechanical relay for power consumption per day, significant power savings will be obtained from the single component of a diode with its inbuilt blocking function and very low maximum voltage drop of $0.9 \mathrm{~V}$ for every 6 A flowing through each blocking diode. The use of relay as compensator may however be the choice component if very many photovoltaics are interconnected for power harness and if power consumption of the mechanical relay is irrelevant or negligible. Relay compensation is also associated with the adverse and ignorable clicking sound from the metallic parts as it makes contacts. The relay requires a $12 \mathrm{~V} \mathrm{DC}$ supply source from the battery terminals that triggers it to operate and turn on connection to load terminals. 


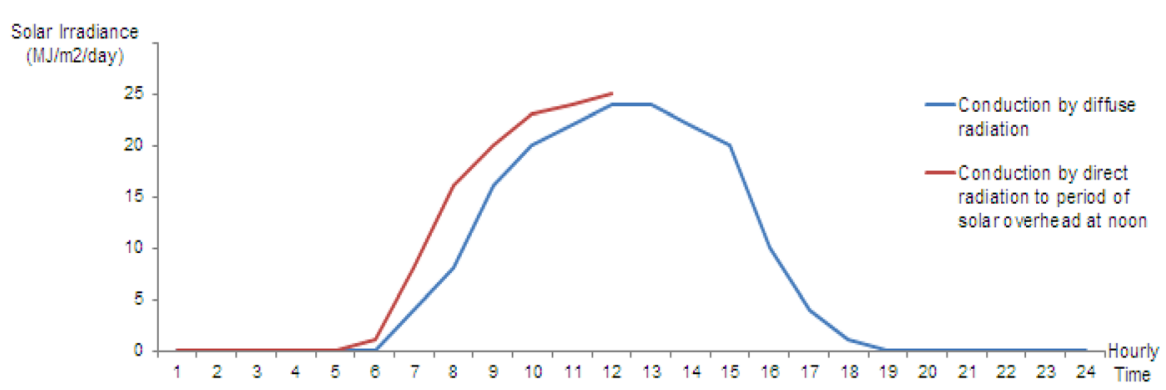

Fig. 4 The daily energy dissipation of the East wing pvAs

\section{Results and discussion}

\subsection{Conduction by PVAs}

As early as an average of 6am, solar intensity is been detected in tropical regions and it increases to a threshold allowing conduction of the photovoltaics (pvA) mounted in the east wing of building to experience both direct and diffuse radiation of solar intensity. This conduction was observed to cause a loss of power to the least path of resistance of the other participating but yet to receive solar radiation photovoltaics of the west and front wings, i.e. pvB and pvC thus necessitating addition of blocking diodes. Observance of the trajectory of solar irradiance for the east winged photovoltaics for both the diffused and directed solar radiation effects on generated power was discovered to initiate power production as early as dawn for a normal sunny day. This steady increase in power continued sharply to till late mornings (till overhead solar irradiance) when only diffuse solar irradiance continue to produce appreciable power leading to the energy dissipation shown in Fig. 4. However, on cloudy days, significant drops in the magnitude of generated power were observed and sometimes no power was generated by the pvAs during extreme cloudy conditions. However, a sudden improvement in the weather condition from cloudy to sunny caused a corresponding increase in the magnitude of generated instantaneous power. Sustained solar irradiance (measured in $\mathrm{MJ} / \mathrm{m}^{2} /$ day) created the bulk of needed solar energy requirement for rising solar intensity to power consumer needs.

\subsection{Conduction by PVBs}

As solar irradiance continue to increase towards noon appreciable energy that is enough to cause forward biasing of the diode compensation of pvB (that was in blocking mode) into conduction and power contribution occurs with mostly diffused solar irradiance. On normal sunny days, intermittent direct solar irradiance also accompanies this energy contribution from $8 \mathrm{am}$ as shown in Fig. 5 on the graph. This daily solar irradiance from direct solar radiation ceases for this set of photovoltaics abruptly on cloudy days or days with low solar intensities. Normal sunny days allowed for maximization of solar intensities that included intermittent direct solar 


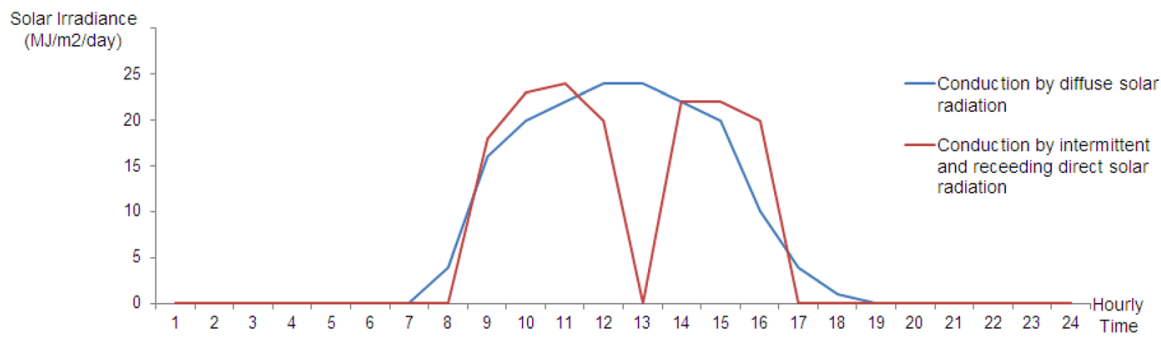

Fig. 5 Daily energy dissipation of the front or North wing pvBs

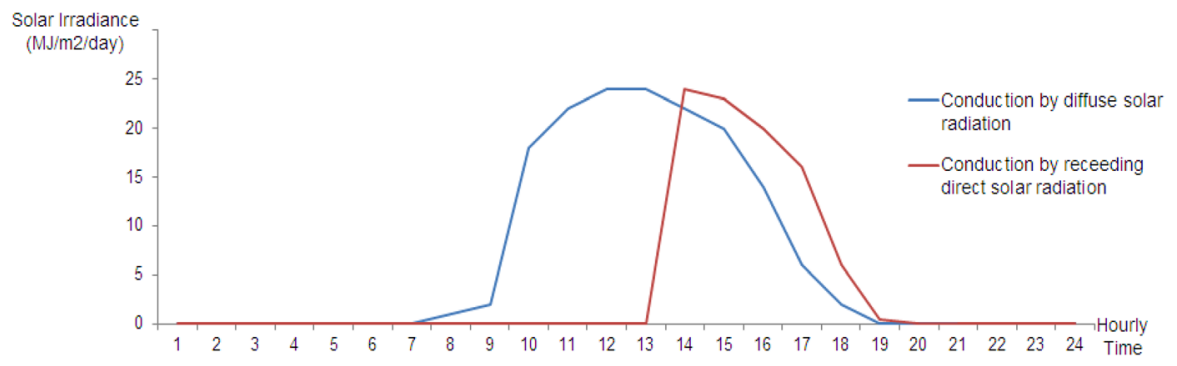

Fig. 6 Daily energy dissipation of the West wing pvCs

radiation as experienced by pvBs and the diffused radiation being experienced up to the end of diffuse solar irradiance for that daily solar intensity. However, experiments show that while the diffuse solar radiation component is constant for the period of solar intensity per day, the direct component of solar radiation is based on spontaneous and non-linearity of the sun's position at that time relative to test site. This is because the sun's intensity was observed to meander over its expected fixed point with respect to earth location or observation site. Also on adverse cloudy days the magnitude of generated power from both modes is significantly reduced over that period up until when a change in weather condition occurs. During the period of loss of direct solar irradiance, diffused radiation is at its maximum and power contribution to consumer terminals is sustained by mostly diffused radiation component as pvCs are still yet to conduct from direct solar irradiance and pvAs are completely cut off from direct solar radiation. The duration of complete loss of direct solar radiation for the participating pvBs was observed to fluctuate around one hour in normal sunny days and more in cloudy days. Also, the direct solar radiation component ended around 4:30pm when the sun has fully receded into the west.

\subsection{Conduction by PVCs}

Also, Fig. 6 shows the daily power generation of the photovoltaics mounted on the wall of the east wing pvCs. From the solar irradiance now reaching that side after noon and up until the last rays from solar irradiance as twilight approaches it 

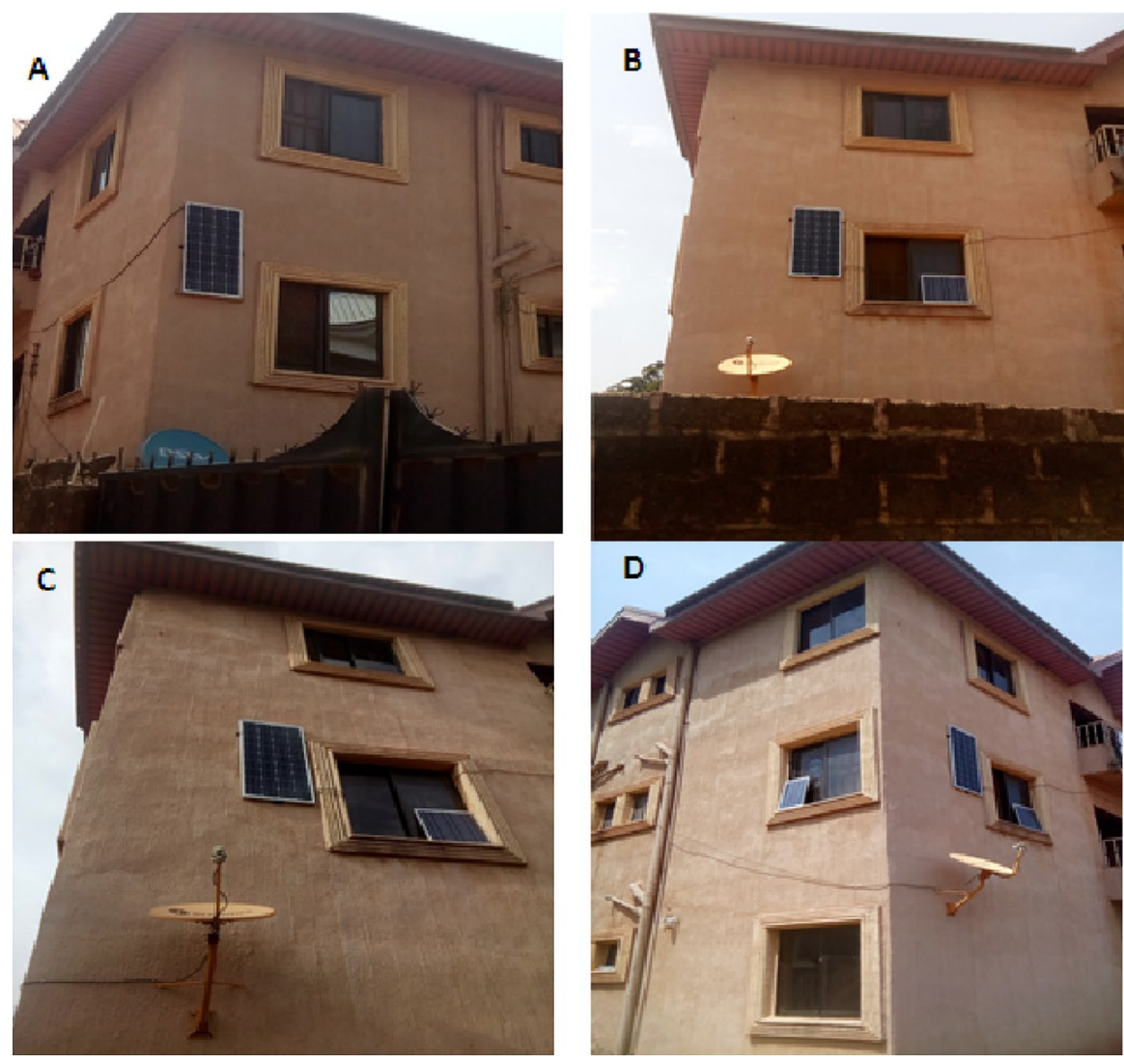

Fig. 7 Pictures of testing site for A East wing pvAs, B Front or North wing pvBs and D West wing pvCs and also showing pvBs receiving only diffused radiation

was observed that sustained power to consumers load terminals was maintained in the resulting energy optimization system. This is due to the blocking diode receiving significant power from diffuse radiation through the pvCs up to a threshold of around 10am to allow for diode conduction. As diffuse radiation reach optimality, pvCs begin to conduct and contribute to consumer terminals. At around 2pm, pvCs begin to experience increased power supply due to receding direct solar irradiance now hitting the west wing photovoltaics and continued diffused radiation. This period is identified as a sharp rise in direct solar radiation in Fig. 6 and corresponds to the period of maximum solar power from west wing photovoltaics on account of the sum of direct and diffused radiation from the receding sun's radiation on a normal sunny day. Again, significant loses in the magnitude of generated power was observed during intermittent cloud cover and cloudy days leading to sometimes no power from extreme cloudy conditions.

Figure 7 shows the testing site's distribution of participating photovoltaics in the resulting power optimization scheme. Photovoltaics of the East wing, pvAs 
Table.1 shows pv array and battery capacity, load demands and diode specifications

\begin{tabular}{lllll}
\hline PV array capacity & Battery capacity & Load demands & $\begin{array}{l}\text { Power compensation/ } \\
\text { diode specifications }\end{array}$ & DC-DC conversion \\
\hline $6 \times 100$ watts & $2 \times 200 \mathrm{AHr}$ & 5000 watts & 6A10-T MIC Diode & MPPT \\
$3 \times 100$ watts & $2 \times 100 \mathrm{AHr}$ & 2500 watts & 6A05-T MIC Diode & PWM \\
$>1000$ watts & $>1000 \mathrm{AHr}$ & $>10,000$ & Relay switching & MPPT \\
\hline
\end{tabular}

(depicted as A in Fig. 7) are always the first to conduct on normal sunny days allowing significant energy from rising sun's intensity of both diffused and direct irradiance. This continues up until around noon while blocking diodes for $\mathrm{pvB}$ and $\mathrm{pvC}$ circuits are in their blocking state. As solar intensity increases pvBs, shown as B in Fig. 7 begin to conduct as its blocking diode reaches conduction thresholds from diffused solar irradiance and later from both diffused and direct solar irradiance as shown in C. The average sun intensity is based on the particular period of the year and weather conditions. D in Fig. 7 shows the west wing photovoltaics, pvC experiencing direct solar and diffused solar irradiance sufficient to allow conducting of blocking diode for $\mathrm{pvC}$ to contribute to the resulting power to consumer terminals. $\mathrm{D}$ also shows the photovoltaics, pvBs in the right hand side experiencing no direct solar irradiance.

\subsection{A comparison with the traditional setup}

Traditionally, photovoltaics are mounted on mostly rooftops or slightly inclined horizontal surfaces for direct solar access and maximum harness of solar energy. Due to restrictions to accessing some rooftops and inability of construction surfaces for direct solar mounts, wall-mounts are being proposed. This has been hinged on the ability of photovoltaics to operate from a combination of diffused and direct solar irradiances. As a benchmark, the traditionally rooftops' mounting of photovoltaics will always allow maximum energy harness of pv array capacity when compared to the same capacity of pv array for wall-mounts and same operating requirements and layouts. This is because wall mounts for power harness only experiences mostly diffused solar radiation propagated across its surface area and intermittent direct solar radiation reaching it. The novelty of this work however, is the fact that an alternative for harnessing solar energy has been investigated and found to provide significant power for consumer terminals. This has been achieved by deployment of a power compensation circuitry for avoiding power leaks in the resulting energy optimization system. An $80 \%$ power efficiency have been achieved on normal sunny days by wall mounts only when compared with $100 \%$ efficiency of rooftops mounted photovoltaics used for control experiment. This has been possible by leveraging on enhanced power attaining equipment such as monocrystalline panels and MPPT charge converters. Thus a restricted rooftop is no longer a barrier for harnessing solar energy for small and medium scale consumers and with scalability, any desired power demand can be achieved. Table 1 shows a distribution of pv array and battery capacity, load demands, DC-DC conversion and diode specifications for implementing 
a wall mounting of photovoltaics for an average small/medium scaled consumer and large scale consumer projections. The power compensation enabled by relay switching as against the silicon rectifier diode allows for harnessing larger power from wall mounted photovoltaics with considerable large surface areas of buildings space or wall mounts. An average size of a $100 \mathrm{~W}$ monocrystalline solar panel is $102 \times 67 \times 3.5 \mathrm{~cm}$ and weighs about $8 \mathrm{~kg}$ according to Solar Power Supply [30].

\section{Conclusions}

Significant energy for consumer needs have been acquired from both direct and diffuse solar radiation in the normal movement of the Sun's trajectory as the earth revolves from east to west and in the path of solar irradiance. This study outlined a design and mounting implementation for layout of wall-mounted photovoltaics products to efficiently harness solar power. The resulting prototype system was used to power a medium-scale homestead consuming less than five thousands watts of energy in a daily rhythm of solar presence. Due to the non-linear solar irradiance from wall-mounting of photovoltaics, the use of MPPT solar conversion was used to achieve the DC to DC conversion and secured operation for consumer added loads. Experimental results showed adequate power sustenance for all contributory and participating photovoltaics in supplying or contributing power to connected load terminals during the normal direct daily solar irradiance based on already computed loads versus daily solar intensity with range of variations. The blocking diode compensation circuitry also ensured significant energy savings from yet to conduct photovoltaics. The power dissipation that was found to have leaked into yet to conduct photovoltaics on wall mountings without power compensation in the other wings was made available at consumer terminals. Investigations into solar wall mounts are necessary and continue to help demystify the generation, distribution and usage of the abundant and renewable energy from the sun. The resultant power from wall mounted photovoltaics could be made available to grid based systems from consumer terminals in an integrated and optimized scheme. It is worth reiterating that fluctuations in power supply to consumer terminals was observed due to sudden and instanteneous cloud cover and nonlinear solar irradiance for some days. This can be controlled by dependent on other power supply methods for consistent power supply.

\section{References}

1. Akpabio, L.E., Etuk, S.E.: Relationship between global solar radiation and sunshine duration for onne, Nigeria. Turk J. of Phys. 27, 161-167 (2002)

2. Akpabio, L.E., Udo, S.O., Etuk, S.E.: Empirical correlations of global solar radiation with meteorological data for onne, Nigeria. Turk J. of Phys. 28, 205-212 (2004)

3. Alternative Energy, Tutorials, 2021. Solar Irradiance. https://www.alternative-energy-tutorials. com/solar-power/solar-irradiance.html. Accessed 24 Feb 2021

4. Bauer, W., Westfall, G.D.: University Physics with Modern Physics, vol. 2. McGraw Hill Companies Inc., New York (2011)

5. Becker, S.: Calculation of direct solar and diffuse radiation in Israel. Int. J. Climatol. 21, 15611576 (2001). https://doi.org/10.1002/joc.650 
6. Burgess, P.: (2009). Variation in light intensity at different latitudes and seasons, effects of cloud cover, and the amounts of direct and diffused light. School of Applied Sciences, Cranfield University, Bedfordshire. http://www.ccfg.org.uk/conferences/downloads/P_Burgess.pdf. Accessed 14 May 2017

7. Clean Energy, Reviews, 2021. What is a Solar charge controller. https://www.cleanenergyrevi ews.info/blog/mppt-solar-charge-controllers. Accessed 30 May 2021

8. Diodes Incorporated. 2021. https:/www.digchip.com/datasheets/parts/datasheet/1850/6a10-tpdf. Accessed 19 April 2021

9. Electronic Component Database. Datasheet for the 6A10-T MIC Silicon Rectifier Diode. http:// www.digchip.com/datasheets/parts/datasheet/1850/6A10-T_amp.php. Accessed 9 Sept 2021

10. EnergySage, 2021. Monocrystalline and Polycrystalline Solar Panels: what you need to know. https://news.energysage.com/monocrystalline-vs-polycrystalline-solar/. Accessed 3 Apr 2021

11. Engineering360, 2020. Solar Irradiance. http://www.globalspec.com/learnmore/sensors_trans ducers_detectors/weather_sensing/solar_radiation_instruments. Aaccessed 20 Oct 2020

12. Finsterie, W., Montillet, J.P., Schmutz, W., Sikonja, R., Kolar, L., Treven, L.: The total solar irradiance during the recent solar minimum period measured by SOHO/VIRGO. Sci. Rep. 11, 7835 (2021). https://doi.org/10.1038/s41598-021-87108-y

13. Haigh, J.D.: 2011. Solar Influences on climate. Grantham Institute for Climate Change, Briefing paper No 5

14. Halouani, N., Nguyen, C.T., Vo-Ngoc, D.: Calculation of monthly average global solar radiation on horizontal surfaces using daily hours of bright sunshine. Sol. Energy 50(3), 247-258 (1993)

15. Hughes, E., Hiley, J., Brown, K., Smith, K.I.: 2012. Hughes Electrical and Electronic Technology. 11th Edition. Pearson Education Limited, England

16. Iqbal, M.: An Introduction to Solar Radiation. Academic Press, New York (1983)

17. Illera, P., Fernández, A., Pérez, A.: A simple model for the calculation of global solar radiation using geostationary satellite data. Atmos. Res. 39(1-3), 79-90 (1995)

18. Leonics, 2021. Basics of MPPT Solar Charger Controller. http://www.leonics.com/support/artic le2_12j/articles2_14j_en.php. Accessed 16 Feb 2021

19. Linares-Rodríguez, A., Ruiz-Arias, J.A., Pozo-Vázquez, D., Tovar-Pescador, J.: Generation of synthetic daily global solar radiation data based on ERA-Interim reanalysis and artificial neural networks. Energy 36(8), 5356-5365 (2011)

20. Hussein, E.M.A.: Radiation Mechanics - Principles and Practice. Elsevier Publishing Inc (2007)

21. Makarov, S.N., Ludwig, R., Bitar, S.J.: Practical Electrical Engineering. Springer International Publishing, Switzerland (2016)

22. Morrison, J.C.: Modern Physics for Scientists and Engineers. Elsevier publishing Inc (2010)

23. NASA, 2008. Man-Systems Integration Standard Volume 1. http://msis.jsc.nasa.gov/sections/ Sect. 05.htm\#_5.7_RADIATION. Accessed 4 Febr 2019

24. Okogbue, E.C., Adedokun, J.A., Holmgren, B.: Hourly and daily clearness index and diffuse fraction at a tropical station, Ile-Ife, Nigeria. Int. J. Climatol. 29(8), 1035-1047 (2014)

25. Osinowo, A.A., Okogbue, E.C.: Investigation of the sky optical conditions over selected stations in the major vegetation zones of Nigeria. Int. J. Sustain. Energ. 33(2), 357-366 (2014)

26. Osinowo, A.A., Okogbue, E.C., Ogungben, S.B., Fashanu, O.: 2015. Analysis of Global Solar Irradiance over Climatic Zones in Nigeria for Solar Energy Applications. J. Solar Energy. 1-9

27. Radosavljevic, J., Dordevic, A.: Defining of the intensity of solar radiation on horizontal and oblique surfaces on earth. Work. Living Environ. Protect. 2(1), 77-86 (2001)

28. Solar Magazine, 2020. Monocrystalline vs Polycrystalline Solar Panels. https://www.solarmagaz ine.com/solar-panels/monocrystalline-vs-polycrystalline-solar-panels. Accessed 27 Dec 2020

29. SolarReviews, 2021. Types of solar panels: which one is the best choice? https://www.solarrevie ws.com/blog/pros-and-cons-of-monocrystalline-vs-polycrystalline-solar-panels. Accessed 27 Mar 2021.

30. Solar Power, Supply, 2021. 100Watt solar panel monocrystalline. Available at: https://www.solar powersupply.en/100-watt-solar-panel-monocrystalline. Accessed 10 Sept 2021

31. The International Renewable Energy Agency (IRENA), 2012. Summary for policy makers: renewable power generation costs. http://www.irena.org/publications. Accessed 6 Jan 2017

32. The World Bank, 2020. Solar photovoltaic power potential by country. http://www.worldbank. org/en/topic/energy/publication/solar-phoovoltaic-power-potential-by-country. Accessed 17 Mar 2021 
33. Umoh, M.D., Udo, S.O., Udoakah, Y.N.: Estimating global solar radiation on horizontal surface from sunshine hours over Port Harcourt, Nigeria. J. Electr. Electron. Eng. Res. 6:1-5 (2013)

34. Wind and Sun, 2021. What is Maximum Power Point Tracking (MPPT). http://www.solar-electric. com/learning-center/mppt-solar-charge-controllers.html/\#: :.text=An\%20MPPT\%2 C\% 20or\%20 maximum\%20power,battery\%20bank\%20or\%20utility\%20grid

Publisher's Note Springer Nature remains neutral with regard to jurisdictional claims in published maps and institutional affiliations. 